\title{
Submillimetre polarimetric observations of magnetic fields in star-forming regions
}

\author{
Rachel L. Curran ${ }^{1}$, Antonio Chrysostomou ${ }^{2}$ \\ and Brenda C. Matthews ${ }^{3}$ \\ ${ }^{1}$ School of Cosmic Physics, Dublin Institute for Advanced Studies, \\ 5 Merrion Square, Dublin 2, Ireland \\ email: rcurran@cp.dias.ie \\ ${ }^{2}$ Joint Astronomy Centre, 880 N. A'ohoku Place, University Park, Hilo, Hawaii USA \\ email: Antonio.Chrysostomou@jach.hawaii.edu \\ ${ }^{3}$ Herzberg Institute of Astrophysics, 5071 West Saanich Road, Victoria, Canada \\ email: brenda.matthews@nrc-cnrc.gc.ca
}

\begin{abstract}
Submillimetre imaging polarimetry is one of the most powerful tools at present for studying magnetic fields in star-forming regions, and the only way to gain significant information on the structure of these fields. We present analysis of the largest sample (to date) of both high- and low-mass star-forming regions observed using this technique. A variety of magnetic field morphologies are observed, with no single field morphology favoured. Both the continuum emission morphologies and the field morphologies are generally more complex for the high-mass sample than the low-mass sample. The large scale magnetic field (observed with the JCMT; $14^{\prime \prime}$ resolution) of NGC1333 IRAS2 is interpreted to be weak (compared to the energetic contributions due to turbulence) from the random field pattern observed. On smaller scales (observed with the BIMA array; $3^{\prime \prime}$ resolution) the field is observed to be almost radial, consistent with the polarisation nulls in the JCMT data - suggesting that on smaller scales, the field may be more important to the star formation process. An analysis of the magnetic field direction and the jet/outflow axis is also discussed. Cumulative distribution functions of the difference between the mean position angle of the magnetic field vectors and the jet/outflow axis reveal no correlation. However, visual inspection of the maps reveal alignment of the magnetic field and jet/outflow axis in 7 out of 15 high-mass regions and 3 out of 8 low-mass regions.
\end{abstract}

Keywords. Techniques: polarimetric, submillimetre, stars: formation, stars: magnetic fields.

\section{Magnetic fields in star formation}

Two main aspects of star formation which are still not fully understood are the initial support of the cloud (cloud lifetimes are much longer than originally predicted by theory), and the launching and collimation of the observed jets and outflows emanating from protostars. Whilst immense progress has been made through theoretical work and simulations - which suggest magnetic fields could be important in both of these aspects - observational evidence of these required fields is needed before these models can be corroborated.

There are currently two models for cloud support in star formation, one which relies on magnetic fields - in which strong fields regulate the collapse via ambipolar diffusion, and one which relies on turbulence, where the pressure from turbulent eddies provides the required support to the cloud. Observations of magnetic fields (especially at early stages of star formation) may prove important in ascertaining the cloud support mechanism, whether from magnetic fields or turbulence alone, or a combination of the two. It has been shown that strong fields (compared to the energetic contributions of the turbulence) 


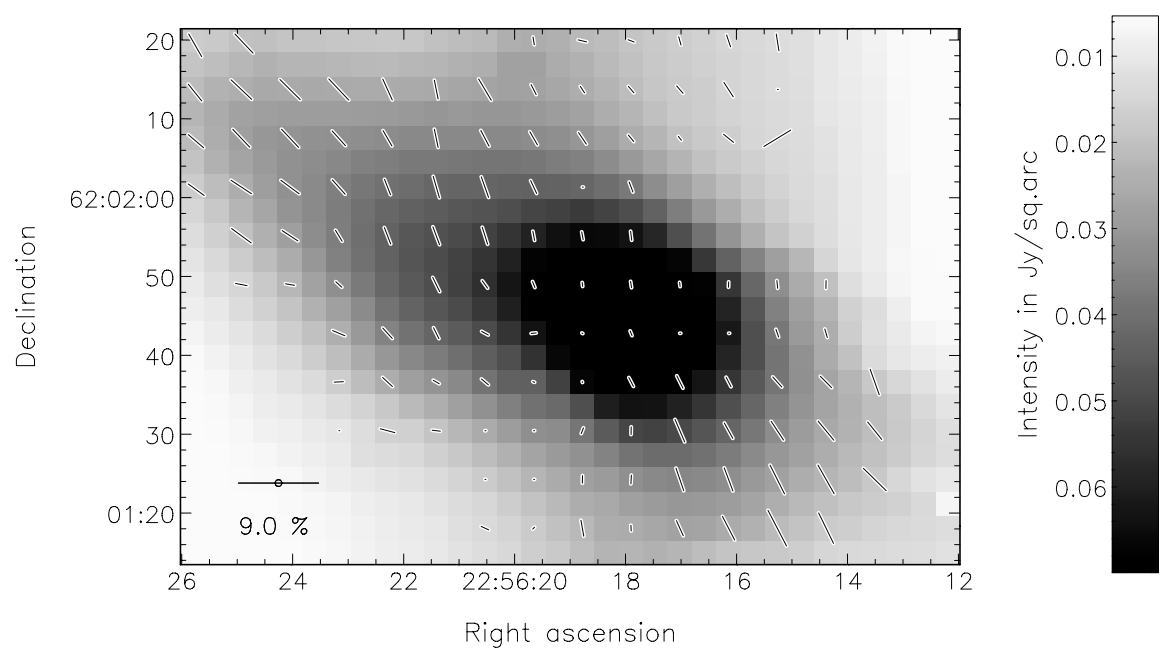

Figure 1. Cepheus A. The greyscale is the $850 \mu \mathrm{m}$ continuum emission, with the B-vectors overlaid to represent the plane-of-the-sky magnetic field component. The field is uniform away from the central core, with a northwest-southeast direction with typical polarisation percentages of $\sim 3-4 \%$. Across the core the polarisation percentage drops to almost zero. There is a largescale east-west outflow in this region, as well as a smaller scale northwest-southeast jet, which aligns well with the magnetic field direction. Epoch J2000.

produce uniform field patterns, whereas weak fields produce more random, disorganised field patterns.

Jet and outflow launching and collimation - according to theory - relies on the magnetic field being $\sim 60^{\circ}$ to the disk plane (for the launching, see the article by J. Ferreira in this volume), and then toroidal in order to collimate the jet. Observations of the direction of the field, both through the disk and along the jet, will help to place constraints on such models. Measuring the magnetic field on different scales may illuminate whether the gas in jets/outflows is dragged along by the magnetic field, or if the magnetic field is dragged along by the gas..

\section{Observing magnetic fields}

There are currently two main approaches to studying magnetic fields in star-forming regions observationally. Zeeman measurements provide the line-of-sight magnetic field strength, although in star-forming regions these estimates come primarily from maser emission and so only reveal the magnetic field for the position of the maser (i.e. from a point source). The second method is polarised thermal emission in the far infrared and submillimetre, which reveals the plane-of-the-sky magnetic field direction throughout the cloud.

Submillimetre polarisation occurs when cool spinning elongated dust grains become aligned to the magnetic field such that their minor axis is parallel to the magnetic field (Davis \& Greenstein 1951). The thermal emission from a volume of such aligned grains is thus polarised, and polarimetry therefore allows for a unique determination of the direction and magnitude of the $\mathbf{E}$-vector from which the $\mathbf{B}$-vector may be inferred. Previous submillimetre polarisation measurements of such star-forming regions have revealed typical polarisation percentages of $<3 \%$ (Matthews \& Wilson 2002, Chrysostomou et al. 2002) although percentages of $<8$ or $9 \%$ have been observed in some star-forming regions (Curran et al. 2004). The millimetre/submillimetre wavelength regime is an ideal 

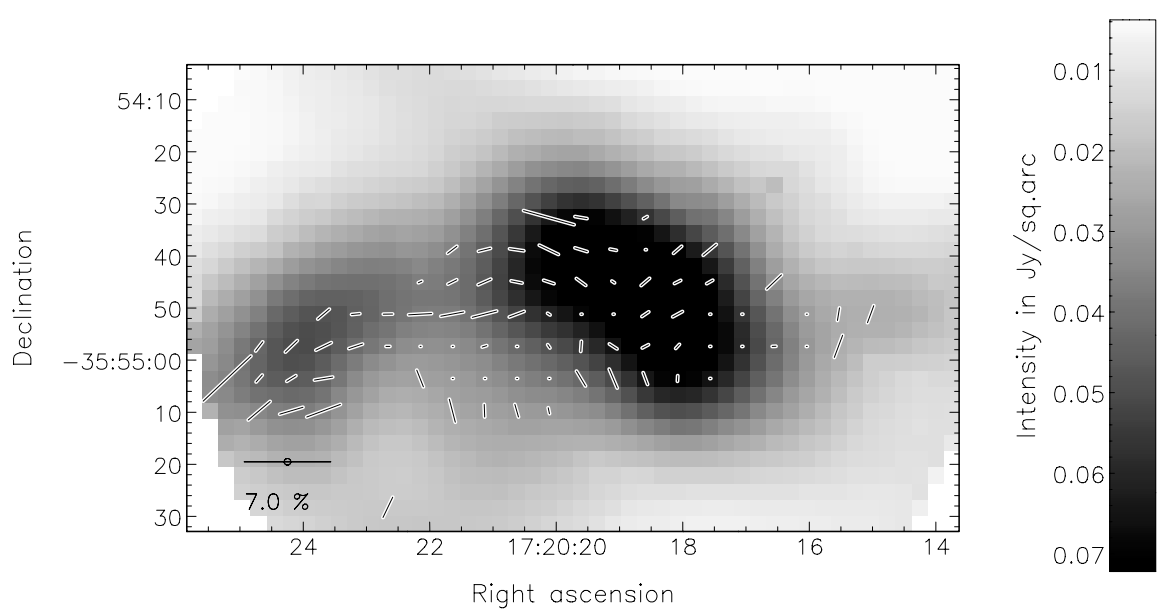

Figure 2. NGC6334A. Greyscale and vectors as for Fig. 1.

probe for star formation as the radiation is optically thin, and therefore is dominated by the densest structures where protostars form. The polarimetry data provide a columnaveraged (and intensity weighted) measurement of the magnetic field direction through the cloud and projected onto the plane-of-the-sky. We have used the Submillimetre Common User Bolometer Array (SCUBA; Holland et al. 1999), in conjunction with the polarimeter (Greaves et al. 2003), on the James Clerk Maxwell Telescope (JCMT) in Hawaii to observe 18 high-mass and 8 low-mass star-forming regions.

\section{Submillimetre emission \& field morphology}

The morphology of the continuum emission is much more varied in the high-mass sample (Curran et al. 2004, Curran \& Chrysostomou 2007a) than in the low-mass sample (Curran \& Chrysostomou, in prep.), as is the morphology of the magnetic fields. The high-mass sample is composed of several (11 out of 18) sources which appear almost spherical (marginally resolved by the JCMT beam), of which 5 of these are associated with fainter, more extended emission. The magnetic field is well traced throughout the extended emission, as well as across the cores. For example, Cepheus A (Fig. 1) consists of a marginally resolved main core and fainter, extended emission detected to its northwest and southeast. The polarimetry reveal that the plane-of-the-sky magnetic field is parallel to this extended emission, in a northwest-southeast direction. Interestingly, the field seems to 'pinch-in', and the polarisation percentage decreases sharply, across the core. This field pattern is consistent with pinched or 'hourglass' field models (Aitken et al. 2002). In this case, the decrease in polarisation percentage across the core would be due to the direction of the magnetic field changing significantly on scales smaller than the JCMT beam, resulting in a lower net polarisation along those lines of sight. Other explanations for the decrease in polarisation percentage with increasing total intensity may be that the grains become more spherical in regions of high density, or that the grains suffer from more collisions, and therefore become un-aligned to the field.

There are also four cores in the high-mass sample that appear elongated, although these could be multiple cores unresolved in the JCMT beam, for example NGC6334A (Fig. 2). The magnetic field of this core is complex, with the polarimetry revealing abrupt changes in direction of the magnetic field. The field appears to be parallel to the extension of gas and dust connecting the main source with the Eastern core, but it then turns 


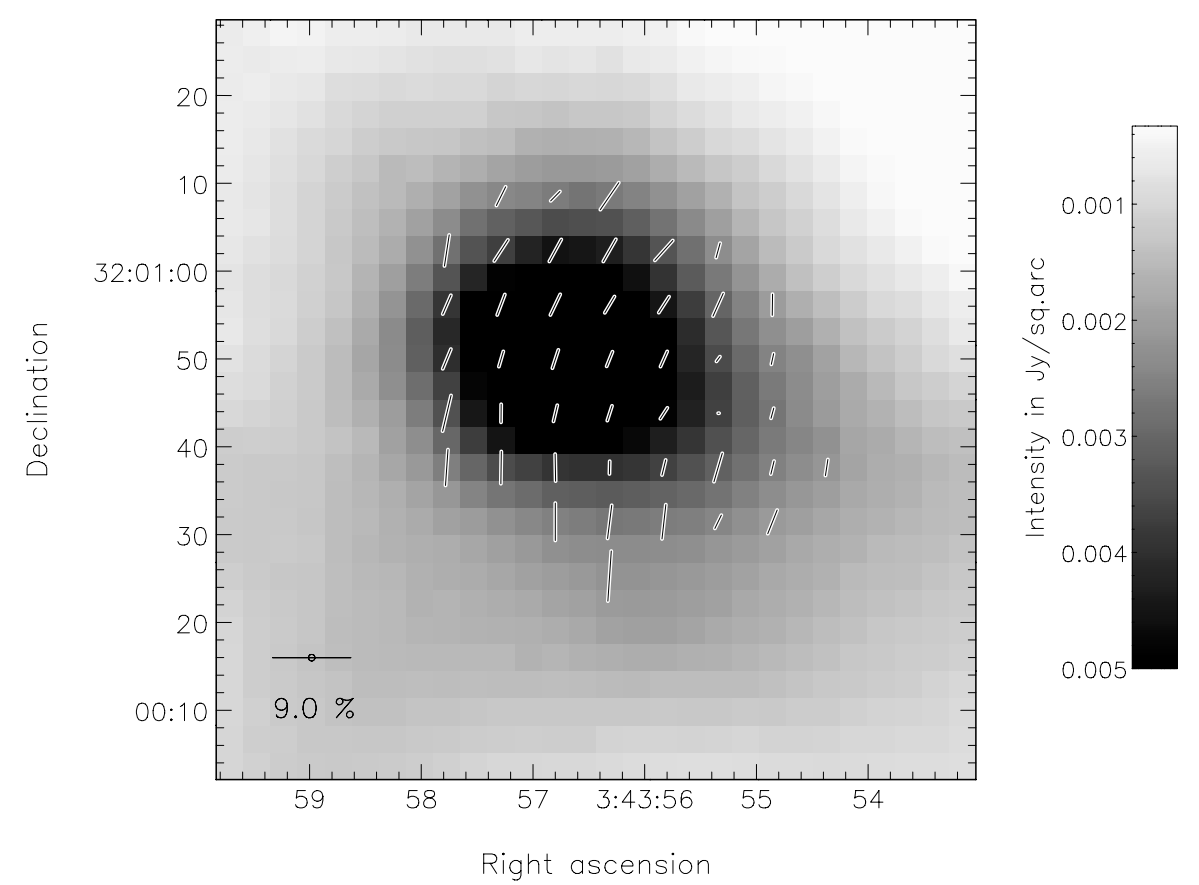

Figure 3. HH211. Greyscale and vectors as for Fig. 1.

perpendicular to the major axis of emission across the southwestern part of the core. There are also several polarisation nulls, some of which are not coincident with regions of high intensity.

The low-mass sample is mainly composed of spherical cores. The magnetic field is generally only traced across the peaks and not in associated extended emission, as these sources are much fainter than the high-mass sources. Even with submillimetre emission similar between sources, we observe a variety of field morphologies. The polarimetry of HH211 (Fig. 3) reveal an ordered field pattern, suggesting a strong magnetic field. The field appears to curve slightly, as it has a north-south direction in the south of the source, curving to become northwest-southeast in the north of the source. The polarisation percentage also remains consistent across the source. In contrast to this ordered field, NGC1333 IRAS2 (Fig. 4) was observed to have an almost random field pattern. There are several polarisation nulls observed across the source. To the north, the magnetic field is roughly north-south in direction, but to the south, the vectors have a large scatter in position angle. This field morphology may indicate a weak magnetic field.

\section{Interferometry}

It has been found in some cases that the magnetic field becomes more important on smaller scales (for example W51, see Chrysostomou et al. 2002 and the higher resolution data of Lai et al. 2001). In order to fully understand the magnetic field of NGC1333 IRAS2, we observed this source with the Berkeley Illinois Maryland Association (BIMA) array, with a resolution of $\sim 3^{\prime \prime}$. The BIMA data are presented in Fig. 5 . The polarimetry trace the magnetic field close in to the protostar, as interferometry 'filters out' the more diffuse emission. The BIMA data agree with the JCMT data -towards the northeast of the peak, the magnetic field is roughly north-south in direction. Moving westwards the 


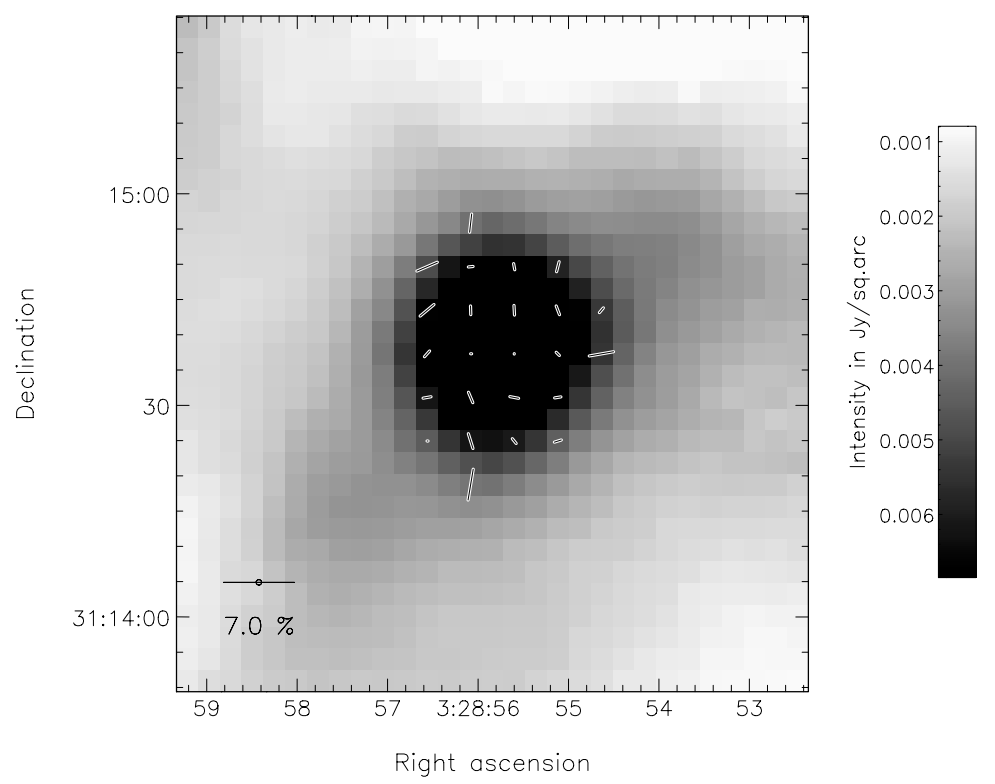

Figure 4. NGC1333 IRAS2. Greyscale and vectors as for Fig. 1.

field then changes to become almost east-west orientated. To the south of the peak, the field is orientated northeast-southwest in direction. This almost radial field at this scale explains the polarisation nulls in the JCMT data - the field direction is significantly different as to cause cancellation of the polarisation within the JCMT beam. At even smaller scales, the magnetic field may have a similar morphology, as once again, the polarisation percentage decreases with increasing intensity.

\section{Magnetic fields and outflows}

Magnetic fields are thought to be important in the launching and collimation of jets and outflows, and so it may be important to compare the observed magnetic field directions to the jet/outflow direction to check for any relationship on these large scales. Previous studies of T Tauri stars (Ménard \& Duchêne 2004) found no relation between the magnetic field and the sample of $\mathrm{T}$ Tauri stars as a whole (both with and without jets), although there was a relation between the magnetic field direction and the $\mathrm{T}$ Tauri stars with jets. A relation between the magnetic field on these scales and the jet/outflow direction may indicate the large scale magnetic field influencing the outflow direction.

The weighted mean of the $\mathbf{B}$-vector position angles were calculated and compared to the position angle of the jet/outflow axis. The magnetic field vectors are not true (i.e. undirectional) vectors. They have a $180^{\circ}$ ambiguity and so range from $0^{\circ}$ through to $180^{\circ}$. The smallest difference between the magnetic field direction and the outflow direction is taken (i.e. if the outflow position angle for the blueshifted lobe is $160^{\circ}$ and the redshifted is $20^{\circ}$, with the magnetic field position angle of $0^{\circ}$, the difference is taken as $20^{\circ}$ as the position angle of the redshifted lobe is used). Fig. 6 shows the cumulative distribution functions for the high-mass sample, low-mass sample, and both samples together. It also shows the function expected for a randomly orientated sample. All three distribution functions are close to the randomly orientated function, and the Kolmogorov-Smirnov test reveals that the high-mass sample has a $84.9 \%$ chance of being randomly orientated, the low-mass $98.8 \%$ and both samples together $99.9 \%$. 


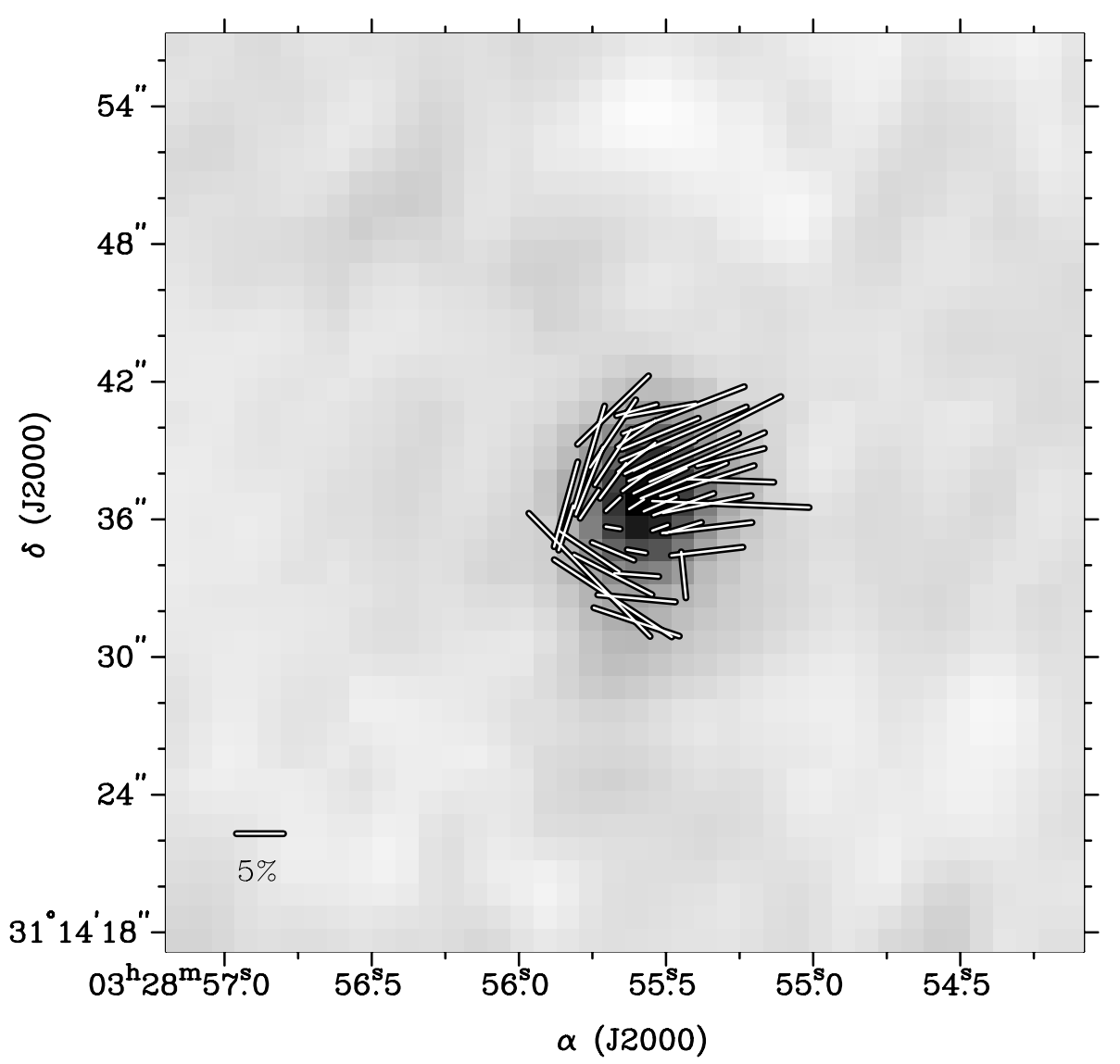

Figure 5. NGC1333 IRAS2 observed with BIMA. The greyscale is the $1.3 \mathrm{~mm}$ continuum emission, with the B-vectors overlaid.

This alignment analysis needs to be treated with caution. For some regions, for example Monoceros R2 (Fig. 7), the mean magnetic field direction may not accurately represent the magnetic field direction. Monoceros R2 has a complex field, which changes direction abruptly. The field is north-south in the north of the region, east-west in the east of the region and northeast-southwest in the southwest of the region. The mean magnetic field direction for this region is $63.5^{\circ}$, or northeast-southwest. Interestingly, the outflow system in this region is also complex, with a large scale north-southwest outflow and a smaller easterly outflow. The above analysis implies there is alignment between the mean magnetic field direction and the southwest part of the outflow, however, visual inspection of the field pattern reveals that there may be alignment between the magnetic field and all three observed directions of the outflows. Visual inspection of the field maps may be a better way of determining outflow alignment.

\section{Conclusions}

We have mapped the plane-of-the-sky magnetic field morphology using submillimetre imaging polarimetry for a sample of 18 high-mass and 8 low-mass star-forming regions. To date, these are the largest samples of this kind. We observe varied field morphologies, with those of the high-mass sample being more complex than those found in the low-mass 


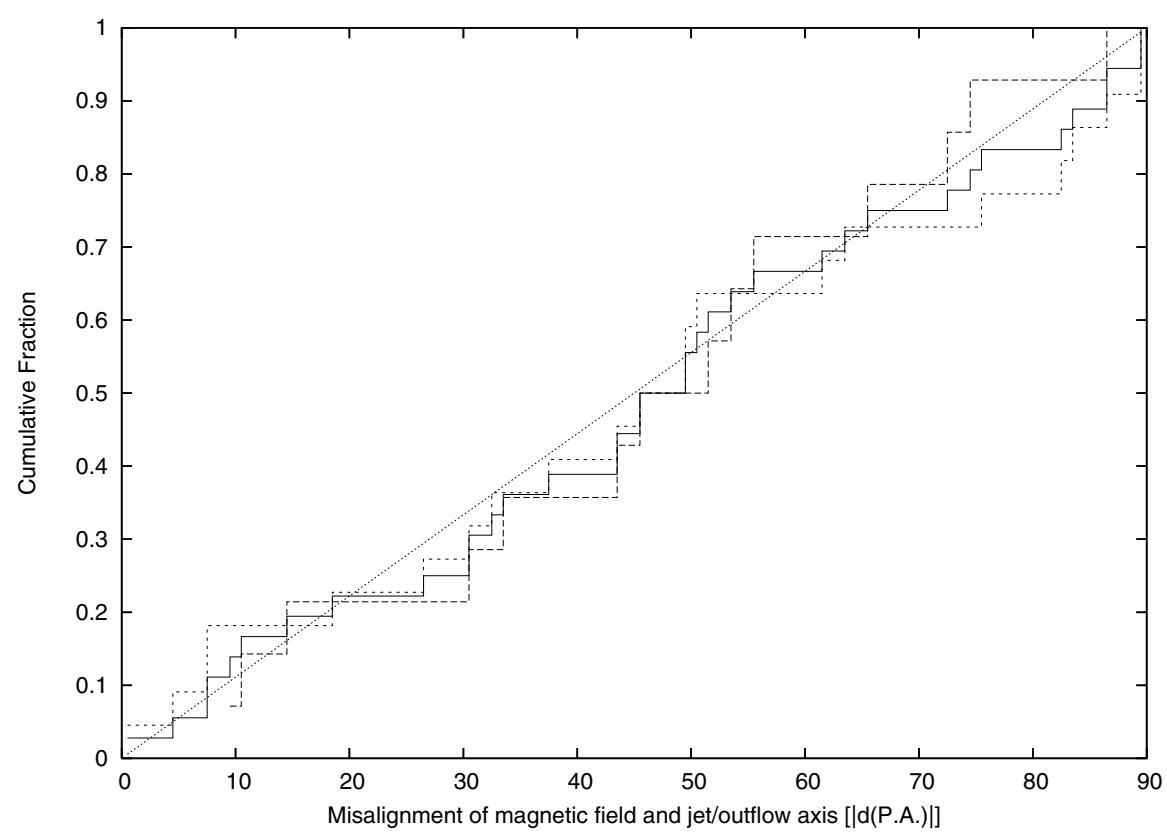

Figure 6. The cumulative distribution function of the difference in PAs between the weighted mean of the magnetic field vectors and the jet/outflow axis. The short-dashed histogram is the high-mass sample, the long-dashed histogram is the low-mass sample. The solid histogram shows the distribution for both samples. The dotted line is the function expected for an infinite randomly orientated sample.

sample. Whilst some of the magnetic field patterns can be interpreted by existing models (e.g. Cepheus A can be compared to the 'hourglass' model from Aitken et al. 2002), others cannot (e.g. Monoceros R2).

Half of the observed sources exhibit decreasing polarisation percentage with increasing total intensity. These may have one of three explanations: either the magnetic field is twisted/changes direction rapidly within the beam of the telescope, the dust grains are more spherical in regions of high density, or the dust grains suffer more collisions in regions of high density and so become unaligned to the magnetic field. All three of these explanations result in a lower net polarisation being measured across the intensity peak.

The JCMT data of NGC1333 IRAS2 has a random field pattern with several polarisation nulls across the source, which may indicate a weak field on these scales. Higher resolution data reveal a field pattern that may be consistent with a radial field morphology. Even at $3^{\prime \prime}$ resolution, there is depolarisation across the core, suggesting the same field morphology may be present at even smaller scales.

Analysis of the mean position angle of the magnetic field vectors and the jet/outflow axis reveal that neither the high- or low-mass samples show significant alignment. Kolmogorov-Smirnov tests reveal that there is a $98.8 \%$ chance that the low-mass sample is randomly orientated, $84.9 \%$ for the high-mass sample and $99.9 \%$ chance that both samples are randomly orientated. The mean magnetic field vectors' position angle may not be the best way of summarising the magnetic field direction within such regions, as we observe several sources in which there are abrupt changes in direction of the magnetic field (see for example Monoceros R2, Fig. 7). For regions where the magnetic field direction changes abruptly, modal averages (in reasonable sized bins) may be more 


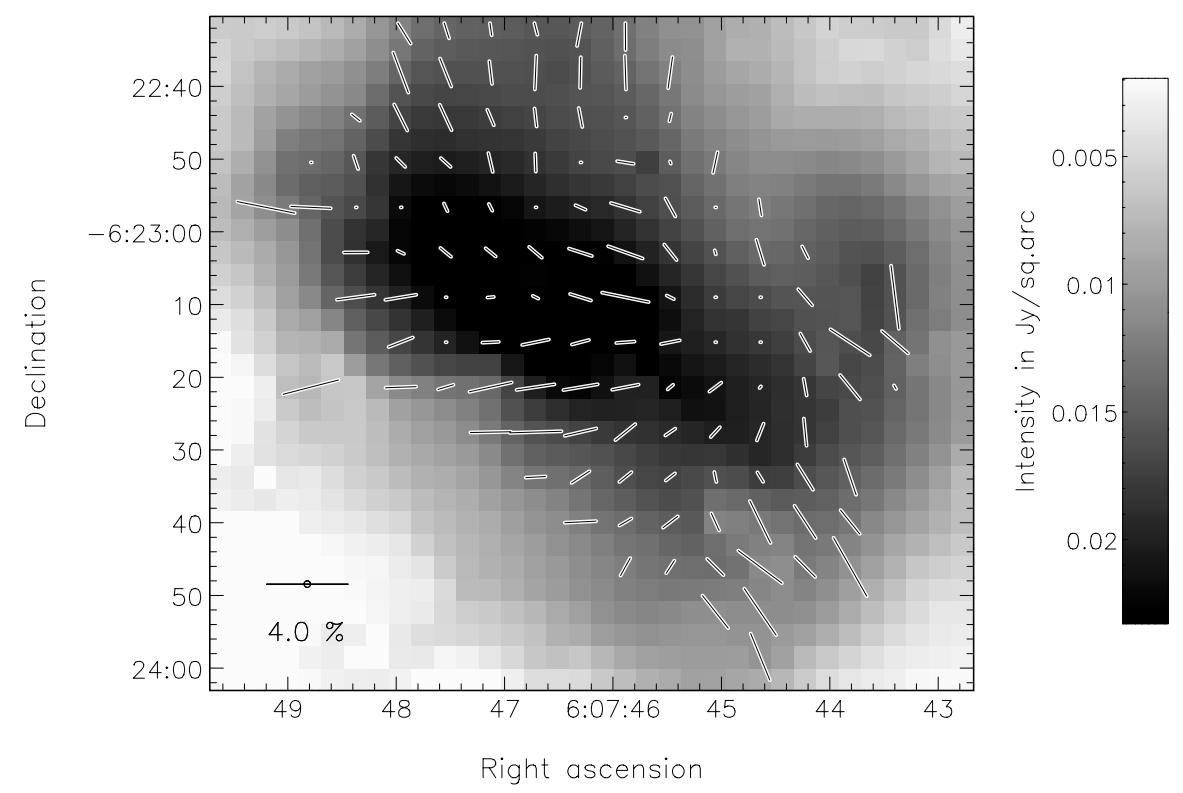

Figure 7. Monoceros R2. Greyscale and vectors as for Fig. 1.

representative. Also, visual inspection of the polarimetry maps is a good way of analysing the alignment.

\section{Acknowledgements}

The James Clerk Maxwell Telescope is operated by The Joint Astronomy Centre on behalf of the Science and Technology Facilities Council of the United Kingdom, the Netherlands Organisation for Scientific Research, and the National Research Council of Canada. The BIMA array was operated with support from the National Science Foundation under grants AST-02-28963 to UC Berkeley, AST-02-28953 to U. Illinois, and AST-02-28974 to U. Maryland. R. L. C. is supported by SFI under grant number 04/BRG/P02741.

\section{References}

Aitken, D.K., Estathiou, A., Hough, J.H. \& McCall, A. 2002, MNRAS 329, 647

Chrysostomou, A., et al. 2002 A\&A 385, 1014

Curran, R.L., Chrysostomou, A., Collett, J.L. et al. 2004, A\&A 421, 195

Curran, R.L. \& Chrysostomou, A. 2007a, MNRAS Submitted.

Curran, R.L. \& Chrysostomou, A. 2007b, in prep.

Davis, L. \& Greenstein, J.L. 1951, ApJ 114, 206

Greaves, J.S., et al. 2003 MNRAS 340, 353

Holland, W.S., et al. 1999 MNRAS 303, 659

Lai, S.-P., et al. 2001, ApJ 561, 864

Matthews, B.C. \& Wilson, C.D. 2002 ApJ 571, 356

Ménard, F. \& Duchêne, G. 2004, A\&广A, 425, 973 